\title{
Decoding the microvesicle message
}

Microvesicles are shed from the membranes of many types of cell and can transfer their contents to other cell types. Those shed by tumour cells have been implicated in promoting tumour progression by manipulating the surrounding environment. Now, Johan Skog and colleagues have shed light on how microvesicles from glioblastoma cells achieve these effects.

The authors initially characterized the RNA that was contained in the microvesicles shed by cultured glioblastoma cells: a broad range of mRNA and microRNA, but not rRNA, was observed, and over 2,200 mRNA transcripts were enriched more than fivefold (up to 380-fold) in the microvesicles as compared with the donor cells. Ontology analysis of the most abundant transcripts showed that mRNAs connected to angiogenesis, cell proliferation, immune response, cell migration and histone modification were present at high levels in microvesicles. Fluorescently labelled microvesicles were seen to be internalized into endosome-like structures by both brain endothelial cells and primary glioblastoma cells. The authors went on to demonstrate that the mRNA in these microvesicles can be translated in the recipient cells.

To investigate the processes that can be mediated by these microvesicles, the authors looked at the effect of glioblastoma microvesicles on human brain microvascular endothelial cells in an in vitro angiogenesis assay. They found that microvesicles caused similar levels of tubule elongation compared with angiogenic growth factors. Assessment of the expression levels of angiogenic proteins in microvesicles and in donor cells revealed that the three most enriched proteins in the microvesicles were angiogenin, interleukin 6 (IL-6) and IL-8, which have been connected with increased angiogenesis and malignancy of gliomas. In addition, glioblastomaderived microvesicles caused an eightfold increase in growth of cultured glioma cells over 3 days, compared with the fivefold increase observed for controls. These results indicate that the microvesicles that are released by glioblastoma cells can stimulate angiogenesis in brain endothelial cells and the proliferation of glioma cells, revealing both environment-modifying and self-promoting effects.

So, as these microvesicles are present in the serum of patients with glioblastoma, can their RNA content profile be exploited as a biomarker for the tumours? Expression of a variant of the epidermal growth factor receptor (EGFR) mRNA - EGFRvIII defines a clinical subtype of glioma. The variant transcript was detected in 14 of 30 tumour samples, which is consistent with previous findings, and in 7 of 25 samples of microvesicles isolated from patient serum. Moreover, EGFRvIII mRNA was not detected in serum samples taken 2 weeks after resection of EGFR $v I I I^{+}$ tumours, supporting the assumption that the microvesicles originate from the tumour. This less invasive method of assessing the EGFRvIII status of tumours might have clinical utility, as $\mathrm{EGFRvIII}^{+}$gliomas may respond better to treatment with EGFR inhibitors than those that have normal EGFR. Further work is warranted to assess the potential of RNA profiling of serum microvesicles to monitor different diagnostic markers in glioblastoma and other cancer types. Isobel Barry

ORIGINAL RESEARCH PAPER Skog, J. et al. Glioblastoma microvesicles transport RNA and proteins that promote tumour growth and provide diagnostic biomarkers. Nature Cell Biol. 16 Nov 2008 (doi:10.1038/ncb1800)

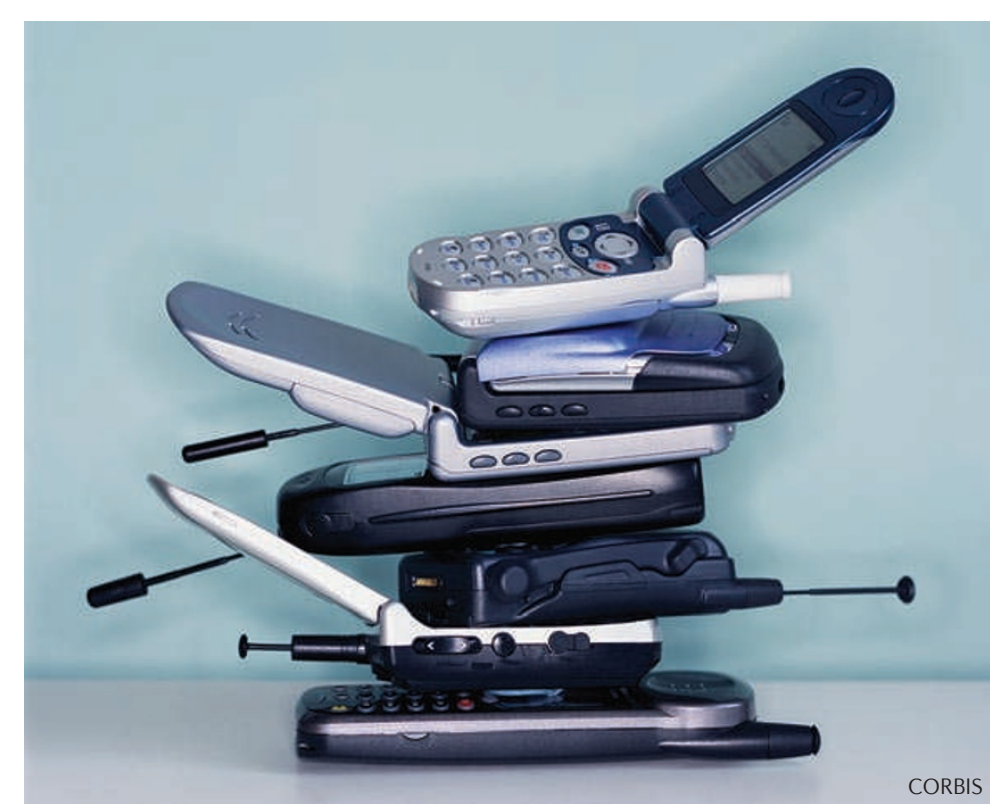

